\title{
Coal Ash as Flame Retardants and Smoke Suppressants for Flexible Poly (Vinyl Chloride) ${ }^{1}$
}

\author{
Juan-juan Zhao ${ }^{1, a}$, Chun-guang Song ${ }^{1, b}$, Yin-qiu Wei ${ }^{1, c}$, Ming Gao ${ }^{1, d}$ \\ ${ }^{1}$ School of Environmental Engineering, North China University of Science and Technology, Box 206, \\ Yanjiao Beijing 101601, China \\ a zhjj@ncist.edu.cn, ${ }^{\mathrm{b}} 1114149912 @ q q . c o m,{ }^{c}$ Weiyinqiu8899@163.com, ${ }^{\mathrm{d}}$ gaoming@ncist.edu.cn
}

Key words: Coal ash, $\mathrm{Sb}_{2} \mathrm{O}_{3}$, Flame retardant, PVC, Cone calorimeter, TG

Abstract. Coal ash as flame retardant was used to flexible poly (vinyl chloride) (PVC), and the flame retardant and smoke suppressant properties of PVC were investigated by the limiting oxygen index, smoke density rating, and cone calorimeter tests (CONE), the thermal degradation behaviors of PVC were studied by thermogravimetric analysis (TG) in nitrogen atmosphere. The CONE result indicated that coal ash can reduce the heat release rate and smoke production rate in flame-retardant PVC. The TG result showed that the sample with coal ash has higher thermal stability than pure PVC. Besides, PVC treated with coal ash and $\mathrm{Sb}_{2} \mathrm{O}_{3}$ showed a high limiting oxygen index, high decomposition temperature, which indicated that the flame retardance of the treated PVC was improved.

\section{Introduction}

In recent years, many types of chemical compounds have been reported as flame retardants and smoke suppressants for flexible PVC, including metal alloys, inorganic compounds [1], coordination compounds, and organic compounds [2]. $\mathrm{Sb}_{2} \mathrm{O}_{3}$ is one of the important inorganic flame retardant, as a synergistic agent added to the flame retardant polymeric materials containing halogen flame retardant, widely used in plastic products, rubber, textile fabric, coating and polymer materials [3]. Because of the high loading it is essential that good degree of flame retardancy be obtained, but mechanical properties decrease obviously. Using coupling agents and synergists are good ways to solving this problem. Many elements, such as alloys, organic substances and inorganic compounds including antimony, tin, zinc, copper, iron and molybdenum, have been used in the flame retardation and smoke suppression of PVC. Coal ash contains many kinds of metal oxides, which may have the similar effective. The purpose of our present study is to study flame retardant and smoke suppressant of the samples treated with combinations of coal ash.

\section{Experimental}

\section{Materials}

PVC (SG2, Beijing chemical factory Co., Ltd); dioctyl phthalate (DOP) (Tianjin east China reagent factory); $\mathrm{Sb}_{2} \mathrm{O}_{3}$ (industrial grade, Shanghai huigu chemical products Co., Ltd); Tribasic lead sulfate, Dibasic lead phosphate, commercially available; Zinc stearate (industrial grade, Tianjin east China reagent factory).

\footnotetext{
${ }^{1}$ Contract grant sponsor: Fundamental research funds for the Central Universities: 3142013102.
} 


\section{Preparation of Flame Retardant PVC Samples}

Formulation according to predetermined material (including PVC, DOP, tribasic lead sulfate, dibasic lead phosphate, zinc stearate, $\mathrm{Sb}_{2} \mathrm{O}_{3}$, coal ash) are mixed in a mixer 3 to 5 minutes at 35 $45^{\circ} \mathrm{C}$. Then the mixture was completely mixed on the two-roll mill at $165^{\circ} \mathrm{C}$ for $6-8$ min, compressed at $180^{\circ} \mathrm{C}$ to form sheets of $100 \mathrm{~mm} \times 100 \mathrm{~mm} \times 3 \mathrm{~mm}$. The compositions of all of the samples are listed in Table 1.

Table 1 The composition of the samples

\begin{tabular}{cccccccc}
\hline Sample & $\begin{array}{c}\text { PVC } \\
(\mathbf{g})\end{array}$ & $\begin{array}{c}\text { DOP } \\
(\mathbf{g})\end{array}$ & $\begin{array}{c}\text { Tribasic } \\
\text { lead } \\
\text { sulfate } \\
(\mathbf{g})\end{array}$ & $\begin{array}{c}\text { Dibasic lead } \\
\text { phosphate } \\
(\mathbf{g})\end{array}$ & $\begin{array}{c}\text { Zinc } \\
\text { stearate(g) }\end{array}$ & $\begin{array}{c}\mathbf{S b}_{2} \mathbf{O}_{3} \\
(\mathbf{g})\end{array}$ & $\begin{array}{c}\text { Coal } \\
\text { ash } \\
(\mathbf{g})\end{array}$ \\
\hline 1 & & & & & & \\
2 & 100.0 & 30.0 & 2.0 & 2.0 & 0.5 & 0.0 & 0.0 \\
3 & 100.0 & 30.0 & 2.0 & 2.0 & 0.5 & 7.0 & 0.0 \\
4 & 100.0 & 30.0 & 2.0 & 2.0 & 0.5 & 6.5 & 0.5 \\
5 & 100.0 & 30.0 & 2.0 & 2.0 & 0.5 & 6.0 & 1.0 \\
6 & 100.0 & 30.0 & 2.0 & 2.0 & 0.5 & 5.5 & 1.5 \\
7 & 100.0 & 30.0 & 2.0 & 2.0 & 0.5 & 5.0 & 2.0 \\
8 & 100.0 & 30.0 & 2.0 & 2.0 & 0.5 & 4.5 & 2.5 \\
9 & 100.0 & 30.0 & 2.0 & 2.0 & 0.5 & 4.0 & 3.0 \\
10 & 100.0 & 30.0 & 2.0 & 2.0 & 0.5 & 3.0 & 4.0 \\
11 & 100.0 & 30.0 & 2.0 & 2.0 & 0.5 & 2.0 & 5.0 \\
\hline
\end{tabular}

\section{Characterization}

The limiting oxygen index (LOI) test was performed with a JF-3 oxygen index test instrument (Jiangning Analytical Instrument Factory, China) in terms of the standard LOI test, ASTM D2863-2000; The mechanical properties were tested according to GB/T 1040.2-2006 standard with a LJ-5000 tensile testing machine (Chengde Experimental Factory); Thermo-gravimetry (TG) was carried out on a HCT-2 thermal analyzer (Beijing Hengjiu Scientific Instrument Factory) under a dynamic nitrogen (dried) atmosphere at a heating rate of $10^{\circ} \mathrm{C} \min ^{-1}$; Cone calorimeter measurements were performed at an incident radiant flux of $50 \mathrm{kw} \mathrm{m}$, according to ISO 5660 protocol. The samples $(100 \mathrm{~mm} \times 100 \mathrm{~mm} \times 3 \mathrm{~mm})$ were laid on a horizontal sample holder.

\section{Results and discussion}

\section{LOI and SDR analysis}

Table 2 LOI and SDR results of the samples

\begin{tabular}{cccccccccccc}
\hline Sample & $\mathbf{1}$ & $\mathbf{2}$ & $\mathbf{3}$ & $\mathbf{4}$ & $\mathbf{5}$ & $\mathbf{6}$ & $\mathbf{7}$ & $\mathbf{8}$ & $\mathbf{9}$ & $\mathbf{1 0}$ & $\mathbf{1 1}$ \\
\hline LOI/\% & 27.6 & 35.6 & 33.9 & 33.7 & 33.7 & 33.8 & 33.8 & 33.9 & 33.4 & 32.5 & 30.2 \\
SDR/\% & 82.7 & 87.9 & 76.8 & 69.2 & 64.1 & 66.7 & 80.7 & 69.8 & 75.6 & 64.7 & 62.3 \\
\hline
\end{tabular}

The LOI data in Table 2 presented a trend of "decay-increase-maximum-decay", with the increase of the coal ash content and the decrease of the $\mathrm{Sb}_{2} \mathrm{O}_{3}$ content in the formulation, and the SDR data decreased to a minimum value and then increased of samples 3-7 and samples 7-9, respectively. It can be seen that the SDR value of sample 5 containing $1.5 \mathrm{phr}$ coal ash decreased 
rapidly from 82.7 to $64.1 \%$ of sample 1 . The SDR values of samples $9-11$ gradually decreased. The results obtained from the LOI tests also showed that samples 3-8 were slightly lower than sample 2 but higher than sample 1. Moreover, the highest LOI value of sample 8 with $3 \mathrm{phr}$ coal ash was $33.9 \%$. These results indicated that the addition of a suitable amount of $\mathrm{Sb}_{2} \mathrm{O}_{3}$ and coal ash could increase the flame-retarding and smoke-suppressing of PVC.

\section{Thermal properties of flexible PVC}

Thermogravimetric analysis is one of the commonly used techniques for rapid evaluation of the thermal stability of different materials and also indicates the decomposition of polymers at various temperatures [4-5].

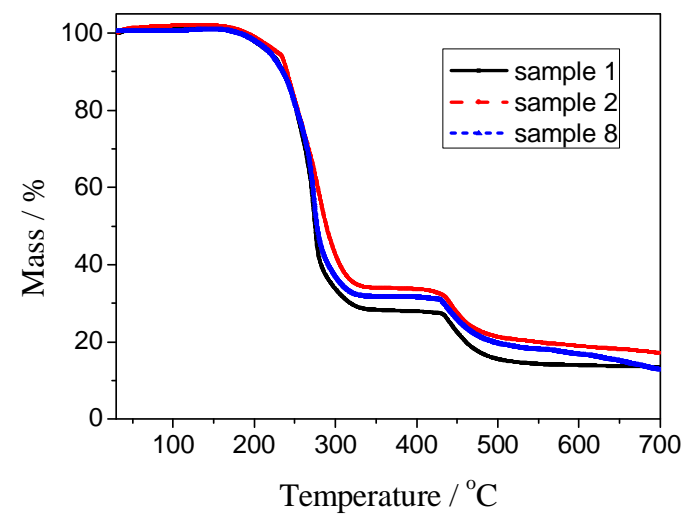

Fig.1 TG curves of samples

It can be seen from Fig. 1 that all the PVC have three main decomposition processes. And the initial decomposition temperatures of them are $218.9,219.9$ and $228.7^{\circ} \mathrm{C}$, respectively, which indicate that pure PVC decompose earlier than that PVC added flame retardant, and the initial decomposition temperatures become shorter with the increase of coal ash content. Additionally, it can be seen from Fig. 1 that the char residues at $700^{\circ} \mathrm{C}$ for sample 1, sample 2 and sample 8 are 12.8, 17.1, and 13.5 mass\%, respectively. The increase of char yields agrees with mechanism of flame retardant. Introduction of flame-retardants leads to more char formed at the expense of flammable volatile products of thermal degradation, thus suppressing combustion and increasing the LOI.

\section{Cone calorimeter test}

Heat release rate (HRR)

The changes in HRR as a function of burning time for different PVC samples are shown in Fig.2. It can be seen that the peak HRR values of sample 1, sample 2 and sample 8 are 97.8, 21.4, and $93.6 \mathrm{~kW} \mathrm{~m}$, respectively. Moreover, the time to peak HRR (tPHRR) of sample 1, sample 2 and sample 8 are 331, 626, $273 \mathrm{~s}$, respectively. The flame retarded PVC with coal ash was earlier by 58 s. To some extent, this was due to catalyze dehydrochlorination in the presence of coal ash. 


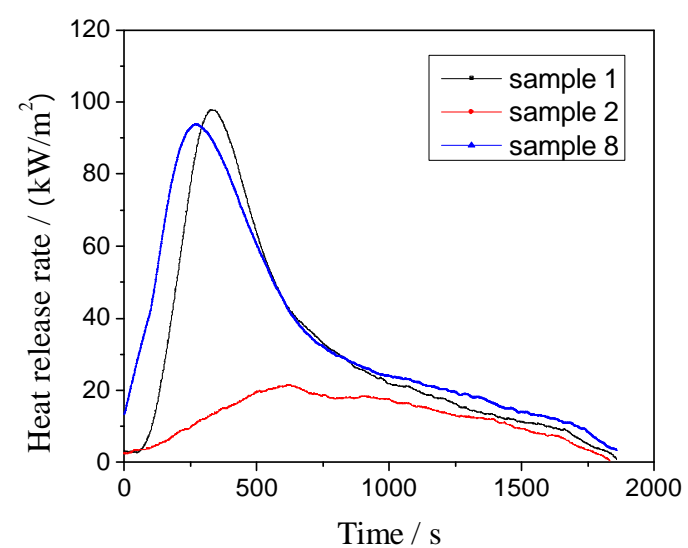

Fig.2 HRR curves of samples

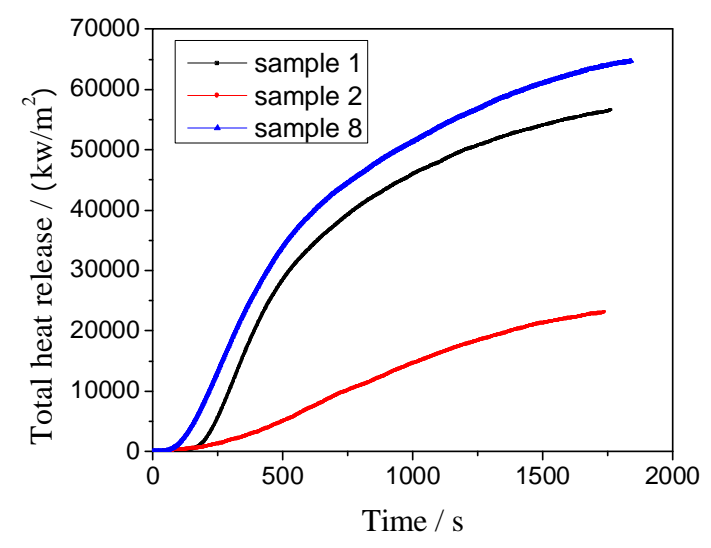

Fig.3 THR curves of samples

Total heat release (THR)

The total heat release (THR) curves of the PVC samples are shown in Fig.3. As we can see, the slope of sample 1 is the biggest one, indicating that the fire spreads of sample 1 is the quickest among all samples. Comparing sample 2 with sample 1, the THR of sample 2 is lower than that of sample 1, which is due to the fact that the char layer of sample 2 is more compact than that of sample 1. These results are probably because the residual char undergoes slow glowing combustion, such that the THR value gradually increases until the end of the test. And the slope of sample 8 is lower but the THR of is slightly higher than that of sample 1.

Smoke production rate (SPR)

As shown in Fig.4, the peak SPR value of sample $1\left(0.178 \mathrm{~m}^{2} \mathrm{~s}^{-1}\right)$ is the largest one among all the samples, the peak SPR value of sample 2 and sample 8 are 0.157 and $0.147 \mathrm{~m}^{2} \mathrm{~s}^{-1}$, respectively, which indicate that the smoke suppression ability of PVC increased with the coal ash as flame retardant added. It can be seen from Fig. 5 that the TSP value of sample 2 and sample 8 are lower than sample 1. Besides, PVC with coal ash produce smoke earlier than sample 1, indicating that it decomposes earlier than sample 1, which is corresponded with HRR and THR curves.

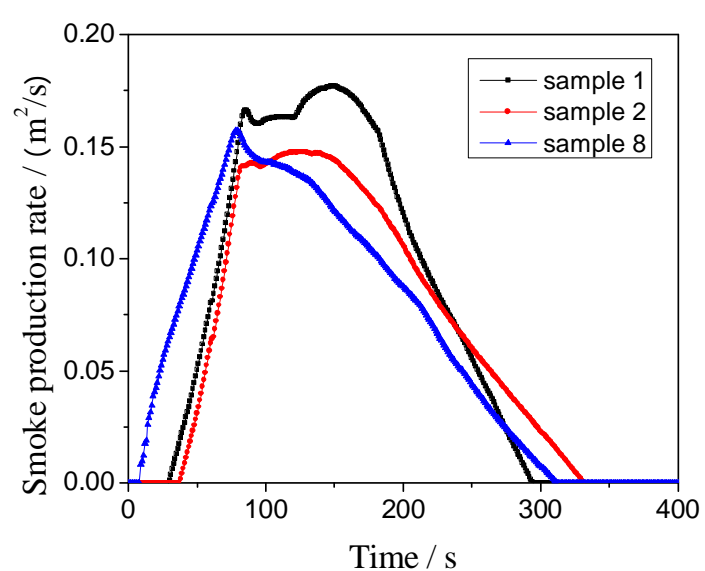

Fig.4 SPR curves of samples

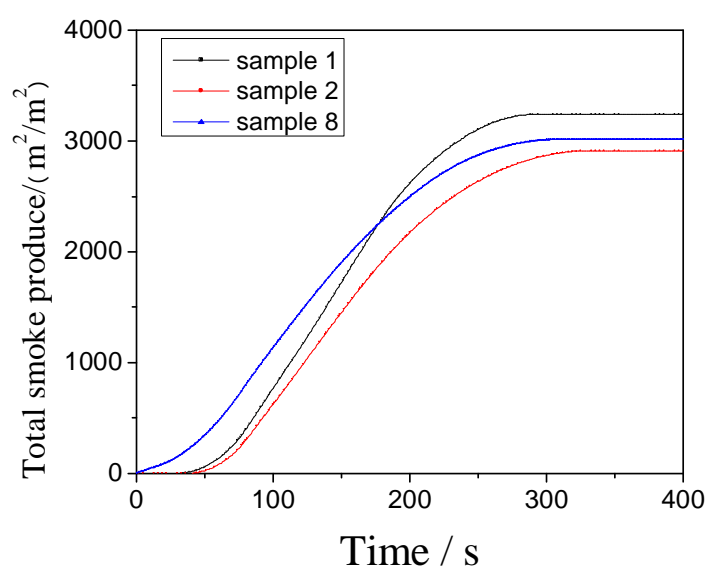

Fig.5 TSP curves of samples

\section{Conclusions}

LOI and SDR studies showed that coal ash had a good flame retarding effect with $\mathrm{Sb}_{2} \mathrm{O}_{3}$, a 
suitable amount of coal ash could greatly increase the LOI and significantly decrease the SDR. Cone calorimeter data show that the addition of coal ash can reduces the heat release rate and smoke production rate in flame-retardant PVC. The TG data show that samples with coal ash have higher char residues than pure PVC, and the initial decomposition temperature become shorter with the increase of coal ash content. Hence, coal ash has the good flame retardant and smoke suppressant effect on PVC.

\section{References}

[1] Qu HQ, Wu WH, Zheng YJ, Xie JX, Xu JZ. (2011).Synergistic effects of inorganic tin compounds and $\mathrm{Sb}_{2} \mathrm{O}_{3}$ on thermal properties and flame retardancy of flexible poly(vinyl chloride). Fire Safety J. 46:462-467.

[2] Deka BK, Maji TK. (2011). Study on the properties of nanocomposite based on high density polyethylene, polypropylene, polyvinyl chloride and wood. Compos Part A.42:686-693.

[3] Porter D, Metcalfe E, Thomas M J K. (2000). Nanocomposite Fire Retardants-a Review [J].Fire and Materials, 24:45-52.

[4] Song YP, Wang DY, Wang XL, Lin L, Wang YZ. (2011). A method for simultaneously improving the flame retardancy and toughness of PLA. Polym Adv Technol. 22:2295-2301.

[5] Wu K, Shen MM, Hu Y. (2011). Synthesis of a novel intumescent flame retardant and its flame retardancy in polypropylene. J Polym Res.18:425-433. 